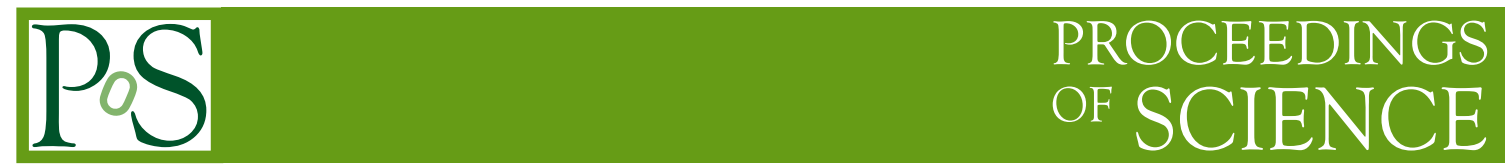

\title{
Recent Results in DIS from Jefferson Lab
}

\author{
David Gaskell* \\ Jefferson $L a b$ \\ E-mail: gaskelldejlab.org
}

\begin{abstract}
Recent results in Deep Inelastic processes measured at Jefferson Lab are presented. In addition to the inclusive reactions typically discussed in the context of Deep Inelastic (electron) Scattering, particular emphasis is given to Deep Exclusive and semi-inclusive reactions. Jefferson Lab has made significant contributions to the understanding of the partonic structure of the nucleon at large $x$, and with its first dedicated measurements is already providing important contributions to understanding the three-dimensional structure of the nucleon via constraints on Generalized Parton Distributions (GPDs) and Transverse Momentum Distributions (TMDs).
\end{abstract}

XVIII International Workshop on Deep-Inelastic Scattering and Related Subjects, DIS 2010 April 19-23, 2010

Firenze, Italy

\footnotetext{
*Speaker.
} 


\section{Jefferson Lab}

Thomas Jefferson National Accelerator Facility (Jefferson Lab) is home to the Continuous Electron Beam Accelerator Facility (CEBAF), an electron accelerator complex capable of beam energies up to $6 \mathrm{GeV}$. The accelerator provides beam to three experimental halls, each originally conceived with different capabilities to provide a fully complementary experimental program.

\subsection{CEBAF}

The CEBAF accelerator at Jefferson Lab is a recirculating linear accelerator with a maximum beam energy of $6 \mathrm{GeV}$. The linacs utilize superconducting RF cavity technology, the first electron accelerator to do so. The electron beam can be accelerated between one and five times through the pair of linear accelerators and can be delivered to up to three halls simultaneously. CEBAF routinely delivers beams with polarization as high as $85 \%$ using a "super-lattice" GaAs photocathode. Beam currents are typically on the order of 10 to $100 \mu \mathrm{A}$ in the "high luminosity" experimental halls (A and C), while Hall B receives beam currents less than $1 \mu \mathrm{A}$.

The CEBAF accelerator will be upgraded in the next few years to a maximum beam energy of $12 \mathrm{GeV}$. In addition, another experimental hall (Hall D) will be built with a dedicated tagged photon beam line.

\subsection{Experimental Halls and Facilities}

The three existing experimental halls at Jefferson Lab each contain their own set of "standard equipment" magnetic spectrometers and detectors for carrying out experiments at Jefferson Lab, although increasingly, each hall uses new, dedicated equipment for specific experiments.

Halls A and C both contain a pair of moderate acceptance, magnetic focusing spectrometers. In Hall A [1], the two identical High Resolution Spectrometers (HRS's) have a solid angle of $\approx 5 \mathrm{msr}$ and momentum acceptance, $d P / P=+/-4 \%$. The Hall A spectrometers can also be augmented with septum magnets to allow very small angle detection of scattered particles. The Hall C spectrometers, the High Momentum Spectrometer (HMS) and the Short Orbit Spectrometer (SOS) boast similar solid angles but larger momentum acceptance $(+/-8 \%$ and -10 to $+20 \%$ respectively), although with slightly worse momentum resolution the HRS's.

Both Halls $\mathrm{A}$ and $\mathrm{C}$ use an array of solid and cryogenic targets at luminosities greater than $10^{38} \mathrm{~cm}^{-2} \mathrm{~s}^{-1}$. Hall A routinely uses a high density polarized ${ }^{3} \mathrm{He}$ target, most recently achieving polarizations of $65 \%$. Hall $\mathrm{C}$ also makes use of polarized targets in the form of dynamically polarized $\mathrm{NH}_{3}$ and $\mathrm{ND}_{3}$ targets. Halls $\mathrm{A}$ and $\mathrm{C}$ have increased their base equipment capabilities by adding large acceptance devices; in Hall A the "Big Bite" spectrometer and in Hall C the Big Electron Telescope Array, BETA.

After the $12 \mathrm{GeV}$ upgrade, Hall $\mathrm{C}$ will replace the SOS with a new, high-momentum spectrometer, the Super-HMS. This spectrometer will have similar properties as the HMS, but with larger maximum central momentum $(11 \mathrm{GeV} / \mathrm{c})$ and smaller minimum angle, $5.5^{\circ}$.

In contrast to the moderate acceptance spectrometers in Halls A and C, Hall B makes use of a large acceptance toroidal spectrometer (the CEBAF Large Acceptance Spectrometer, CLAS [2]). CLAS has almost $2 \pi$ azimuthal coverage over a scattering angle range of $6^{\circ}$ to $140^{\circ}$. The luminos- 
ity in Hall B is limited to $10^{35} \mathrm{~cm}^{-2} \mathrm{~s}^{-} 1$, but its large acceptance allows the measurement of several particles simultaneously over a large region of kinematic phase space.

Hall B will build a new spectrometer for the $12 \mathrm{GeV}$ Upgrade, CLAS12, which will emphasize more forward-angle detection at higher momenta for the purposes of measuring semi-inclusive and deep exclusive processes.

\section{Deep Inelastic Scattering at Jefferson Lab}

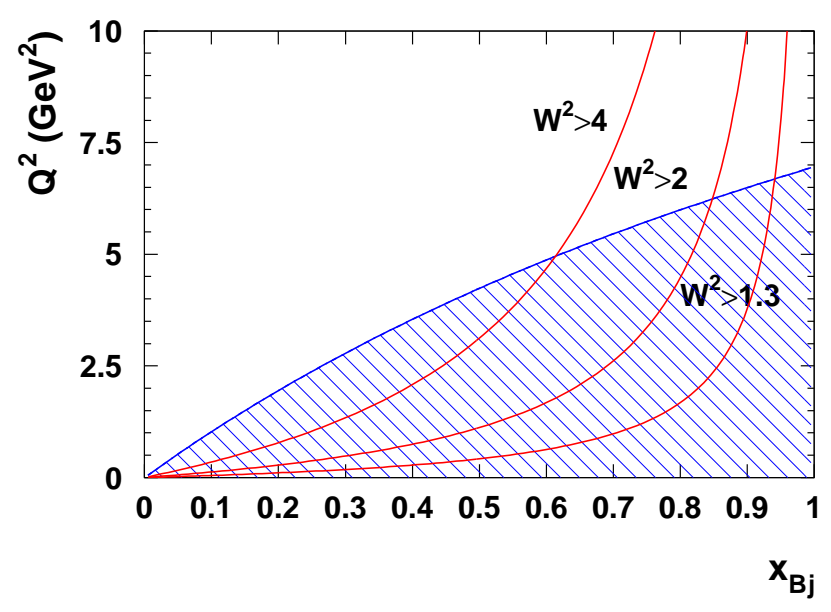

Figure 1: Phase space accessible with the Jefferson Lab $6 \mathrm{GeV}$ beam (hatched). The canonical DIS regime $\left(Q^{2}>1 \mathrm{GeV}^{2}, W>2 \mathrm{GeV}\right)$ is limited to $x=0.6$ due to pure kinematical constraints, while backgrounds limit the low $-x$ limit to about 0.2

The maximum $6 \mathrm{GeV}$ electron beam energy at Jefferson Lab limits the kinematic reach within the canonical DIS regime $\left(Q^{2}>1(\mathrm{GeV} / c)^{2}, W>2 \mathrm{GeV}\right)$ to the region $x=0.2$ to $x=0.6$. Beyond $x=0.6$, the accessible kinematics enter the resonance region (see Fig. 1). At low $x$ the kinematic constraints would allow measurements down to $x=0.1$, however corrections due to radiative effects and charge symmetric backgrounds effectively limit measurements to $x>0.2$. Some Jefferson Lab measurements at $x>0.6$ have been interpreted in the context of partonic degrees of freedom, making use of duality arguments and careful $Q^{2}$-dependence scaling studies.

Within the available phase space, Jefferson Lab has made extensive measurements of inclusive structure functions, both polarized and unpolarized. A great benefit of Jefferson Lab's high luminosity has been the ability to make measurements of relatively low-rate processes like Deep Exclusive reactions and semi-inclusive reactions, in particular measurements of the transversemomentum dependence of these reactions.

It is worth noting that the $12 \mathrm{GeV}$ upgrade will allow measurements within the canonical DIS regime over an $x$-range of $0.1-0.8$. However, the large body of data already taken at large $Q^{2}$ but $W<2 \mathrm{GeV}$ makes it clear that we will likely be able to interpret the $12 \mathrm{GeV}$ data in a partonic framework at even larger $x$. 

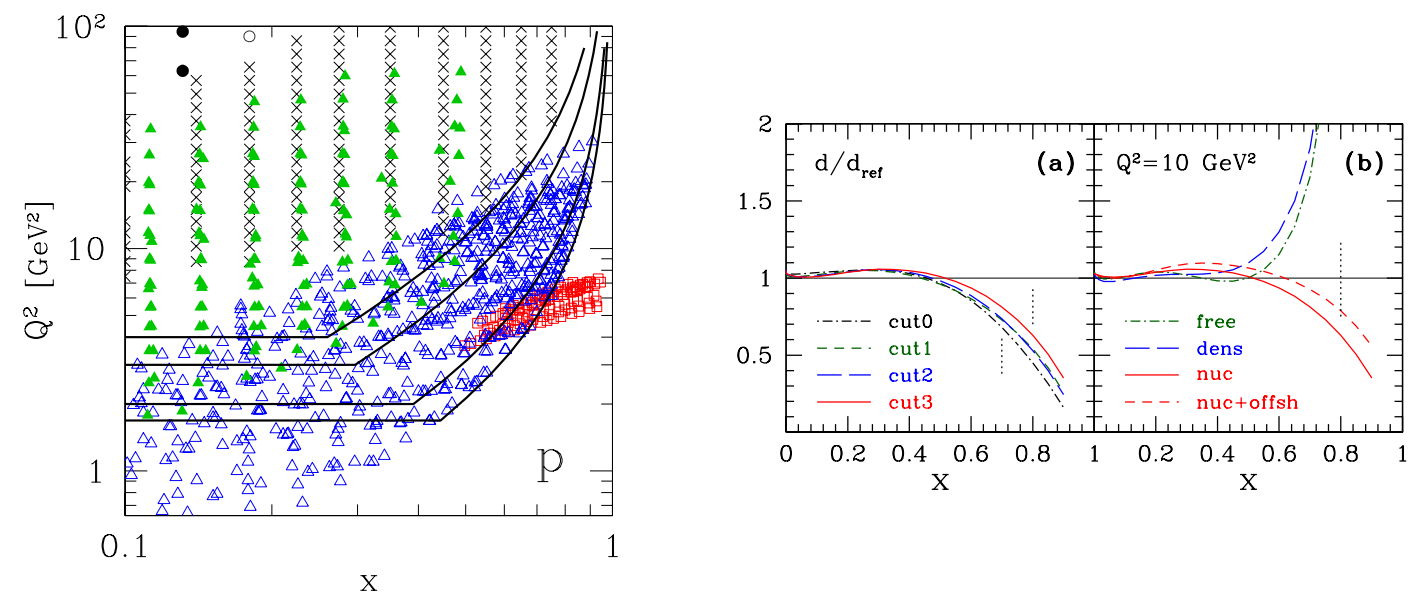

Figure 2: Illustration of new CTEQ fits of Parton Distribution Functions. The plot on the left shows the phase space covered by the available data; NMC (filled green triangles), BCDMS (crosses), H1 (filled black circles), ZEUS (open black circles), SLAC (open blue triangles) and JLab (open red squares). The curves denote different kinematic constraints. The plot on the right shows the variation of the new CTEQ fit for $d(x)$ for the collaboration's various kinematic constraints (left) and when varying the nuclear corrections applied to deuterium data (right).

\section{Inclusive Reactions}

\subsection{Structure Functions at Large $x$}

Despite many years of measurements and study, unpolarized parton distribution fits (MRST, CTEQ) are rather poorly constrained at large values of Bjorken $x$. This is primarily due to rather strict kinematic constraints typically imposed when performing fits to the existing data. For example, in the case of the CTEQ parametrization, the kinematic requirements used $\left(W>3.5 \mathrm{GeV}, Q^{2}>\right.$ $\left.4(\mathrm{GeV} / c)^{2}\right)$ limit the existing "fit-able" data to $x<0.5$. Recently CTEQ has joined forces with Jefferson Lab collaborators to extend their fits to larger $x$ by improving various corrections to unpolarized structure function measurements (target mass correction, nuclear corrections for data from the deuteron), thus allowing one to relax the kinematic constraints noted above [3]. Figure 2 illustrates the results of these attempts. With the improved techniques employed in the PDF ex-

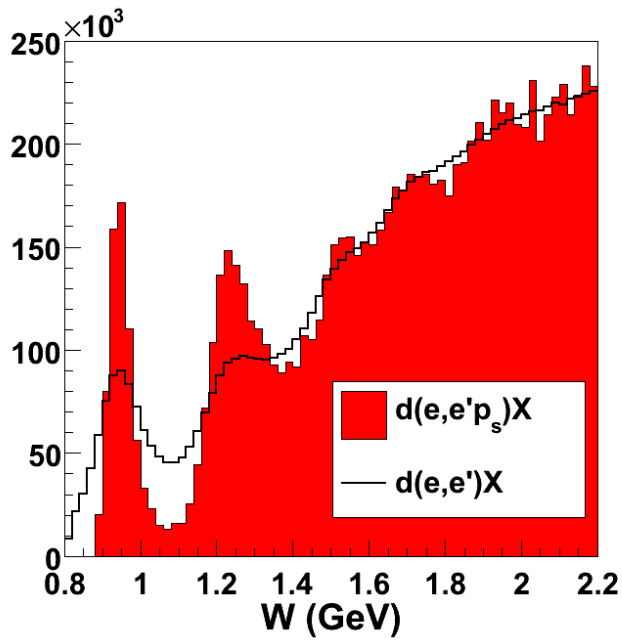

Figure 3: Reconstructed $W$ spectrum in the $D\left(e, e^{\prime}\right) X$ reaction (unfilled histogram) compared to that for the tagged-proton case, $D\left(e, e^{\prime} p_{\text {spec }}\right) X$ from the BONUS experiment in Hall B. traction, the high-precision (albeit low $W$ ) Jefferson Lab data plays an increasingly important role.

The BONUS experiment in Hall B is also aimed at improving our understanding of partonic structure at large $x$. In this case, the experiment aims in particular to reduce the uncertainty due 

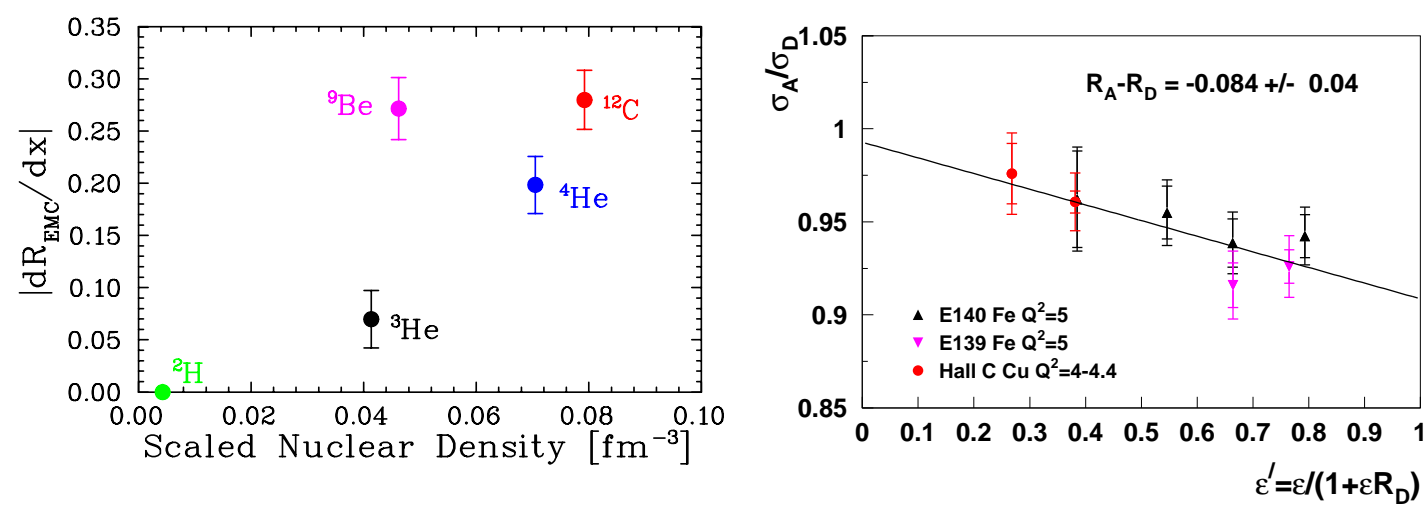

Figure 4: Results from Hall C experiment E03-103. The left plot shows the magnitude of the EMC Effect for $\mathrm{A}=3,4,9$, and 12 vs. nuclear density. The right plot shows E03-103 $\mathrm{Cu}$ results at $x=0.5$ combined with re-analyzed SLAC data (E140) vs. $\varepsilon^{\prime}$; the non-zero $\varepsilon$ dependence implies $R=\sigma_{L} / \sigma_{T}$ is modified in nuclei.

to nuclear effects in the experimental extraction of $F_{2}^{n} / F_{2}^{p}$, from which one can infer the ratio $d / u$ at large $x$. At large $x$, nuclear effects in deuterium become increasingly important. To minimize these effects, the BONUS ("Barely Off-shell NUcleon Structure") experiment uses a radial time projection chamber to tag slow, "spectator" protons at backward angles - detection of these protons implies that a slow, almost on-shell neutron was struck in the $\left(e, e^{\prime}\right)$ reaction. The promise of this technique is shown in Fig. 3. Preliminary results indicate the technique will allow extraction of the structure function ratio up to at least $x=0.6$. A follow-up experiment planned after the $12 \mathrm{GeV}$ Upgrade will extend the kinematic reach to at least $x=0.8$.

\subsection{Nuclear Effects}

Since the original observation of the EMC Effect demonstrated unambiguously that quark distributions are non-trivially modified in nuclei, understanding the source of this modification has been one of the most challenging enterprises in nuclear physics. There are numerous explanations that describe the effect with varying degrees of success, and many experiments have increased the precision and kinematic reach of the available data. At Jefferson Lab, recent measurements in Hall C (Experiment 03-103) were undertaken with the goal of providing new precise data for few-body nuclei $\left({ }^{3} \mathrm{He}\right.$ and $\left.{ }^{4} \mathrm{He}\right)$ and at large $x$, where conventional nuclear effects like Fermi motion are thought to dominate. The results [4] are shown in the left panel of Fig. 4. Here the slope of the $x$ dependence of the ratio $\sigma_{A} / \sigma_{D}$ is shown plotted vs. nuclear density for $A=3,4,9$, and 12 . With the exception of Beryllium, the results are consistent with the naive expectation that the EMC Effect should scale with nuclear density. However, if one considers that the nuclear structure of Beryllium can be considered to be that of a cluster of two $\alpha$ particles with an extra neutron, it makes some intuitive sense that the EMC Effect for ${ }^{9} \mathrm{Be}$ should be more similar to that for ${ }^{4} \mathrm{He}$, i.e., the EMC effect is dominated by the local nuclear environment rather than the average nuclear density.

An additional result from the same experiment is shown in the right panel of Fig. 4. In this case, data from a large $Z$ target $(\mathrm{Cu})$ have been combined with re-analyzed data from SLAC E140. The main improvement here is that effects from the Coulomb field of the struck nucleus have been accounted for. The result of this combined analysis shows a clear $\varepsilon$ dependence in the ratio $\sigma_{A} / \sigma_{D}$, 
implying that $R=\sigma_{L} / \sigma_{T}$ is modified in a nucleus at large $x$ [5]. The source of this modification is unclear, but implies that at large $x$ it may not be entirely accurate to interpret the ratio of $F_{2}$ structure functions as a "simple" modification of parton distribution functions.

\subsection{Spin degrees of freedom}

Jefferson Lab has produced a wealth of inclusive electron scattering data from polarized ${ }^{3} \mathrm{He}, \mathrm{NH}_{3}$, and $\mathrm{ND}_{3}$ targets. While there have been measurements of the polarized structure functions $g_{1 p}$ and $g_{1 n}$ in the DIS regime, and experiments have recently completed that will add significantly to the available data for $g_{2 p}$ and $g_{2 n}$, the bulk of the JLab polarized target data has been taken for $W<2 \mathrm{GeV}$, i.e., the resonance region. This large body of resonance region data has allowed Jefferson Lab experiments to extract the moments of these structure functions with great precision. An example of this is shown

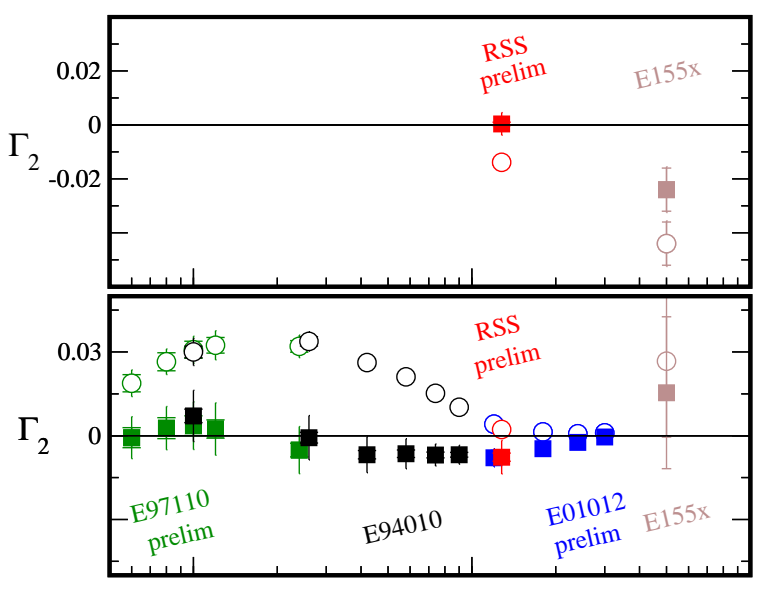

Figure 5: First moment of $g_{2}(x)$ for proton (top) and neutron (bottom). The expectation of the BurkhardtCottingham Sum Rule is verified for all $Q^{2}$ sampled at Jefferson Lab [6].

in Fig. 5. Here, the first moment of the $g_{2}$ structure function, $\Gamma_{2}\left(Q^{2}\right)=\int_{0}^{1} g_{2}\left(x, Q^{2}\right) d x$, has been extracted for both proton [10] and neutron $[7,8,9]$. The so-called Burkhardt-Cottingham sum rule states that this moment should be identically zero for all $Q^{2}$; this expectation is born out by the Jefferson Lab results [6].

It is worth noting that there also exists more, impressive results for the first moment of $g_{1}$ for both proton and neutron. They are not shown here simply for lack of space.

\section{Semi-inclusive Processes}

In the simple, factorized picture of semi-inclusive meson production, the reaction can be visualized as occurring in two steps. The virtual photon produced in the electron scattering process "knocks out" a quark of a particular flavor; this knocked-out quark subsequently hadronizes, forming a quark-antiquark pair (or even a three quark configuration) in the final state. The cross section can be written schematically,

$$
d \sigma \propto \sum_{q, h} f^{H \rightarrow q}(x) d \sigma_{q} D^{q \rightarrow h}(z)
$$

where $f^{H \rightarrow q}$ describes probability to find a quark of a particular flavor in the struck hadron $(H)$, $d \sigma_{q}$ is virtual photon-quark cross section, and $D^{q \rightarrow h}$ describes the probability for the struck quark to hadronize to a particular meson $(h)$. It is easy to see how this picture allows one to attempt to use semi-inclusive processes as a "flavor-tag" for extracting both unpolarized and polarized parton distribution functions. With the addition of transverse degrees of freedom, the above expression becomes,

$$
d \sigma \propto \sum f^{H \rightarrow q}\left(x, k_{T}\right) d \sigma_{q} D^{q \rightarrow h}\left(z, p_{T}\right) .
$$




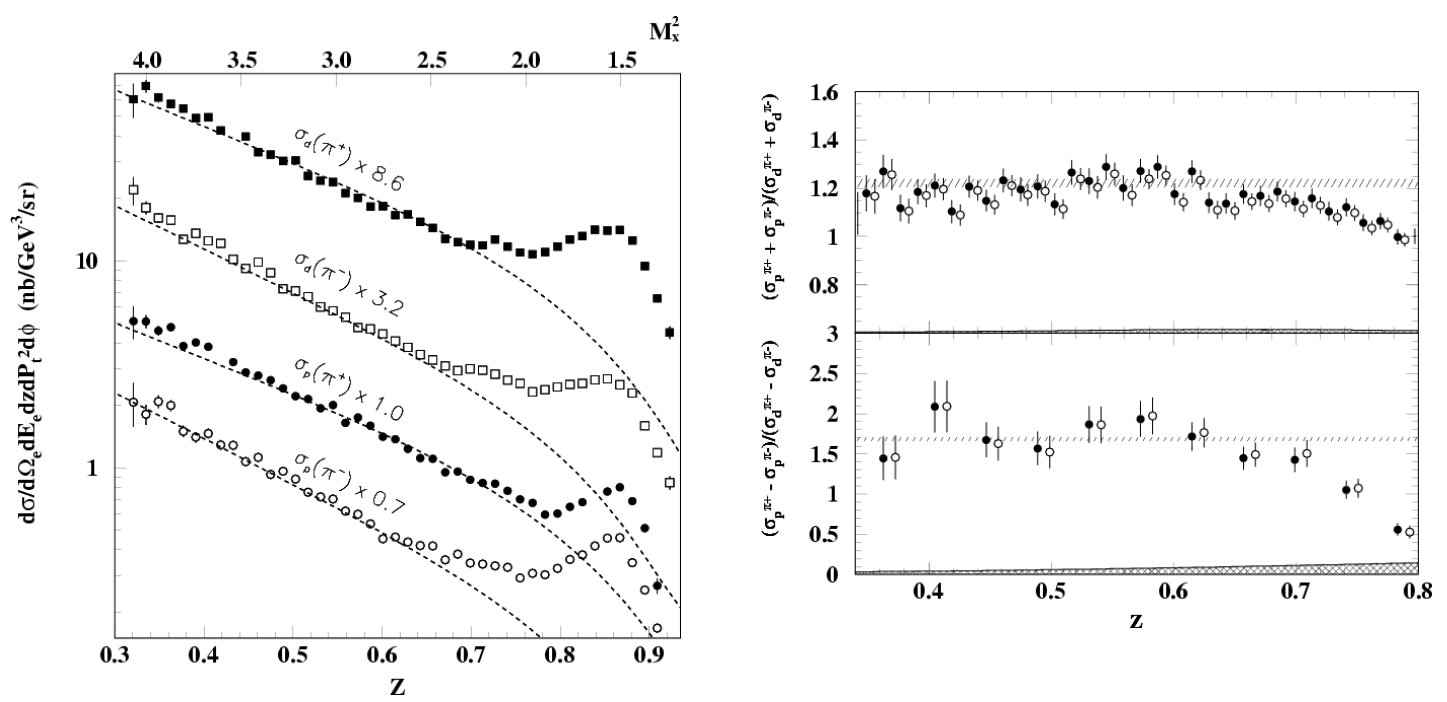

Figure 6: Results from Hall C experiment E00-108 ("Meson Duality"). The cross sections and ratios shown are consistent with a simple, independent factorization picture.

The above implies that by observing the transverse momentum dependence of the outgoing meson $p_{T}$, it is perhaps possible to learn about the intrinsic transverse momentum $\left(k_{T}\right)$ of the quark knocked out of the struck hadron.

Given the relatively low beam energy available at Jefferson Lab, it might seem challenging to use semi-inclusive processes to extract real information about hadronic structure, however results in both the polarized and unpolarized sector suggest that the naive factorization picture described above may apply fairly well.

Experiment E00-108 (the so-called "Meson Duality" experiment) in Hall C measured the $e+A \rightarrow e^{\prime}+\pi+X$ reaction for both $\pi^{+}$and $\pi^{-}$from hydrogen and deuterium targets [11]. The $z$-dependence of the unpolarized cross sections at fixed $x$ were extracted, as well as various combinations of charge and target ratios. The results are shown in Fig. 6; the plot on the left shows the extracted cross sections compared to a calculation that employs fits to PDFs and fragmentation functions from high energy data and assumes the simple factorization hypothesis. The agreement for $z<0.7$ is quite good and only breaks down when the missing mass of the undetected system approaches the mass of the $\Delta$ resonance. The plot on the right shows the proton to deuteron ratio for the sum and difference of charged pion yields. Again, the results are consistent with simple factorization expectations.

The same experiment also measured the $p_{T}$ dependence of the reaction at fixed $x$. Using the combination of $\pi^{+}, \pi^{-}$, and proton and deuteron targets allows one to independently determine the average transverse momentum of the quarks in the nucleon. While the extraction involves several assumptions, a first attempt of this extraction was performed in Ref. [12].

The CLAS collaboration (Hall B) has also used semi-inclusive pion production to glean new information about quark intrinsic transverse momentum [13]. In this case, the CLAS collaboration measured semi-inclusive charged and neutral pion production from a longitudinally polarized proton target. Fig. 7 (left) shows the double-spin asymmetry $\left(A_{1}\right)$ at fixed $x$ and $z$ as a function of the transverse momentum of the outgoing pion. Assuming a simple Gaussian behavior for the 

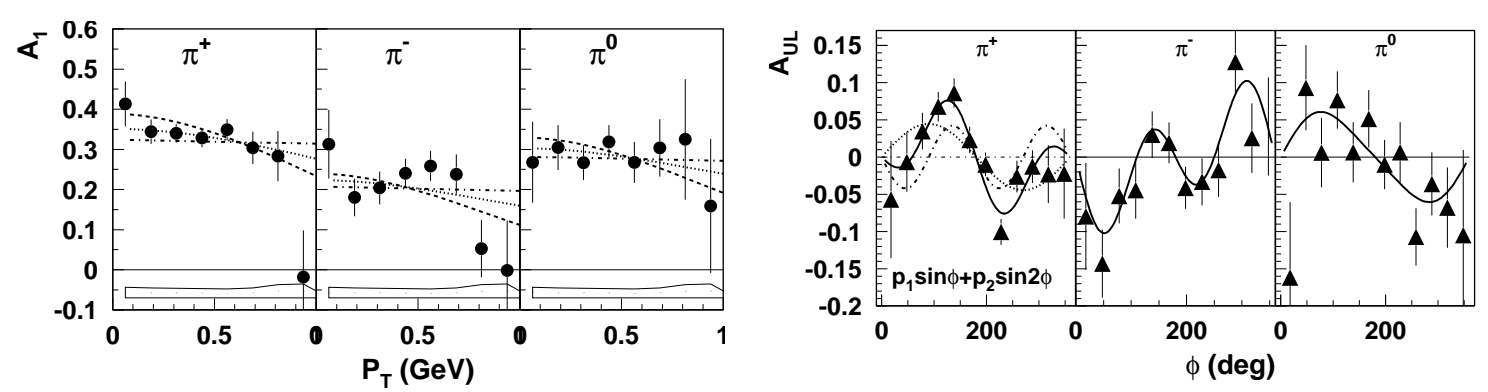

Figure 7: CLAS (Hall B) results for double-spin and single-spin asymmetries from a polarized proton target for semi-inclusive pion production [13]. The plot on the left shows the transverse momentum dependence of $A_{1}$; the fact that the asymmetry is not constant indicates a difference between the transverse momentum dependence of the polarized and unpolarized quark distribution functions. The right plot shows the azimuthal dependence of the single-spin asymmetry. The $\sin 2 \phi$ moment should be sensitive to the $h_{1 L}^{\perp}$ TMD. The $\sin 2 \phi$ moments are non-zero for both $\pi^{+}$and $\pi^{-}$production.

transverse momentum dependence of the quark structure functions and fragmentation functions, the asymmetry can be written [14],

$$
A_{1} \propto \frac{\sum_{q} e_{q}^{2} g_{1}^{q}(x) D(z)}{\sum_{q} e_{q}^{2} f_{1}^{q}(x) D(z)} e^{-z p_{t}^{2} \frac{\mu_{0}^{2}-\mu_{2}^{2}}{\left(\mu_{D}^{2}+z^{2} \mu_{0}^{2}\right)\left(\mu_{D}^{2}+z^{2} \mu_{2}^{2}\right)}},
$$

where $\mu_{0}^{2}\left(\mu_{2}^{2}\right)$ describes the intrinsic transverse momentum of the unpolarized (polarized) quark distribution and $\mu_{D}^{2}$ describes the transverse momentum dependence of the fragmentation function. The fact that $A_{1}$ changes with $p_{T}$ can be explained if the unpolarized quark distribution function $f_{1}$ has a broader $k_{T}$ dependence than the polarized distribution function, $g_{1}$.

The CLAS data can also be used to extract information about a new class of parton distribution, the so-called Transverse Momentum Distributions (TMDs). Extraction of the $\sin 2 \phi$ moment of the azimuthal dependence of the polarized target single-spin asymmetry in semi-inclusive pion production should be proportional to the $h_{1 L}^{\perp}$ distribution function (the distribution of transversely polarized quarks in a longitudinally polarized nucleon). These azimuthal asymmetries are shown in Fig. 7 (right). It is worth noting that the results shown here are from data collected in 2001. More recent data collected in 2009 will increase the statistical precision of these measurements dramatically.

\section{Deep Exclusive Processes}

Generalized Parton Distributions (GPDs) provide yet another window into the three-dimensional structure of the nucleon. GPDs provide the link between Deep Inelastic inclusive processes that provide access to the polarized and unpolarized forward parton distribution functions and elastic processes that provide information on the shapes of hadrons via form factors. The total angular momentum of the nucleon can be related to GPDs via the Ji Sum Rule, while Fourier transforms of the GPDs allow access to quark distributions in impact parameter space.

In recent years, Jefferson Lab has begun a program of dedicated measurements of Deep Exclusive processes (exclusive photon and meson production at large $W$ and $Q^{2}$ ) with the aim of 

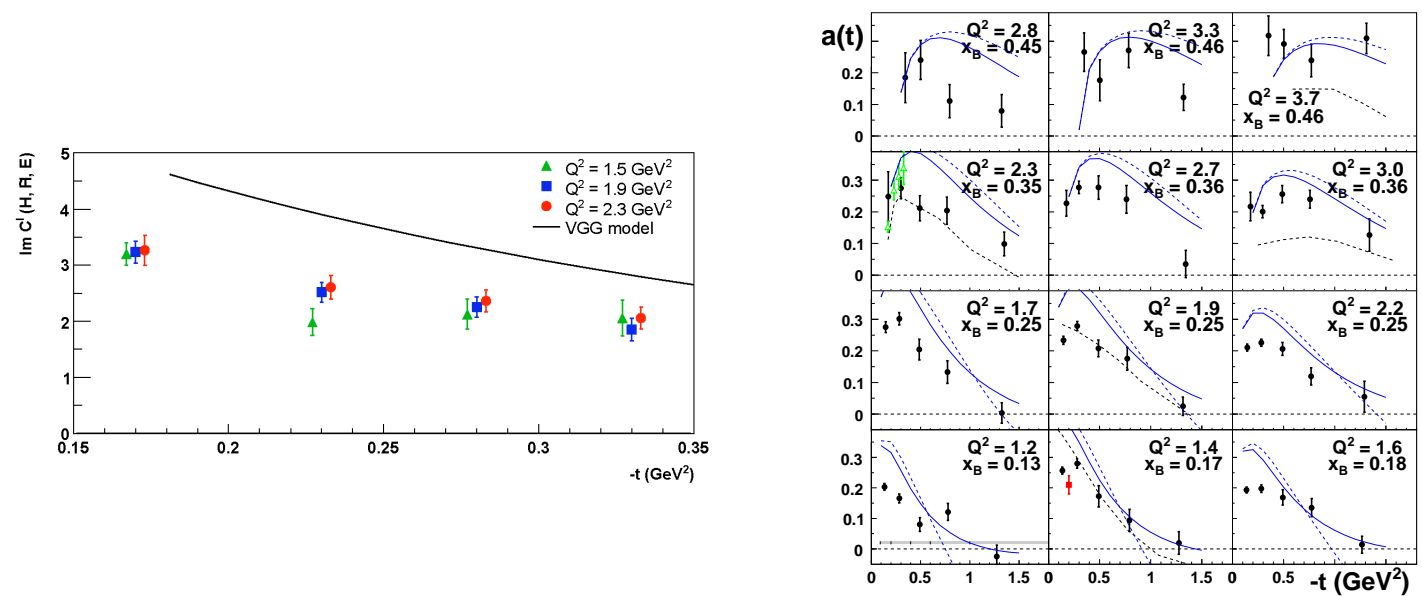

Figure 8: Measurements of the Beam Spin Asymmetry in DVCS. The plot on the left shows results from Hall A; the $Q^{2}$ independence of the extracted Compton Form Factors suggests that even at $6 \mathrm{GeV}$, soft-hard factorization may already hold. The results on the right are from Hall B. The kinematic breadth of the data allows for rigorous tests of models of the DVCS reaction.

extracting information about Generalized Parton Distributions. A major aim of the physics program after the $12 \mathrm{GeV}$ upgrade is to build on these measurements, expanding the kinematic reach and breadth of processes measured.

\subsection{Deeply Virtual Compton Scattering}

A major thrust of the Jefferson Lab Deep Exclusive program has been measurements of Deeply Virtual Compton Scattering (DVCS). Dedicated measurements have been performed in both Halls A and B. Hall B, with its large acceptance spectrometer (CLAS) allows the measurement of a very large phase space in a single experiment, while the Hall A apparatus allows measurements at very high precision at particular kinematic settings.

Results from both halls are shown in Fig. 8. Both plots show results from measurement of the $\sin \phi$ dependence of the Beam Spin Asymmetry (the asymmetry from polarized electrons and unpolarized proton target). The plot on the left shows the Hall A measurement of the twist-2 Compton form factors (CFFs), $\operatorname{Im} C^{I}$, which are a function of the GPDs $H, \tilde{H}, E$ and the nucleon elastic form factors [15]. The apparent $Q^{2}$ independence of the CFFs indicates that, even at JLab energies, we are in a regime where soft-hard factorization, a prerequisite for accessing information about GPDs, seems to hold. The plot on the right shows results for $a(t)$, the $\sin \phi$ amplitude of the Beam Spin Asymmetry as measured in Hall B [16]. Each panel represents a different value of $x$ and $Q^{2}$, spanning the range $\left(x, Q^{2}\right)=(0.18,1.6)$ to $(0.45,2.8)$. Note also the large range of $-t$ sampled.

In addition to the Beam Spin Asymmetry, Hall B has also made measurements of the Target Spin Asymmetry [17], with a new round of data taken in 2009 that immensely increase the statistics available. Hall A also has extracted the Beam Spin Asymmetry for the neutron using data from proton and deuteron [18]. 

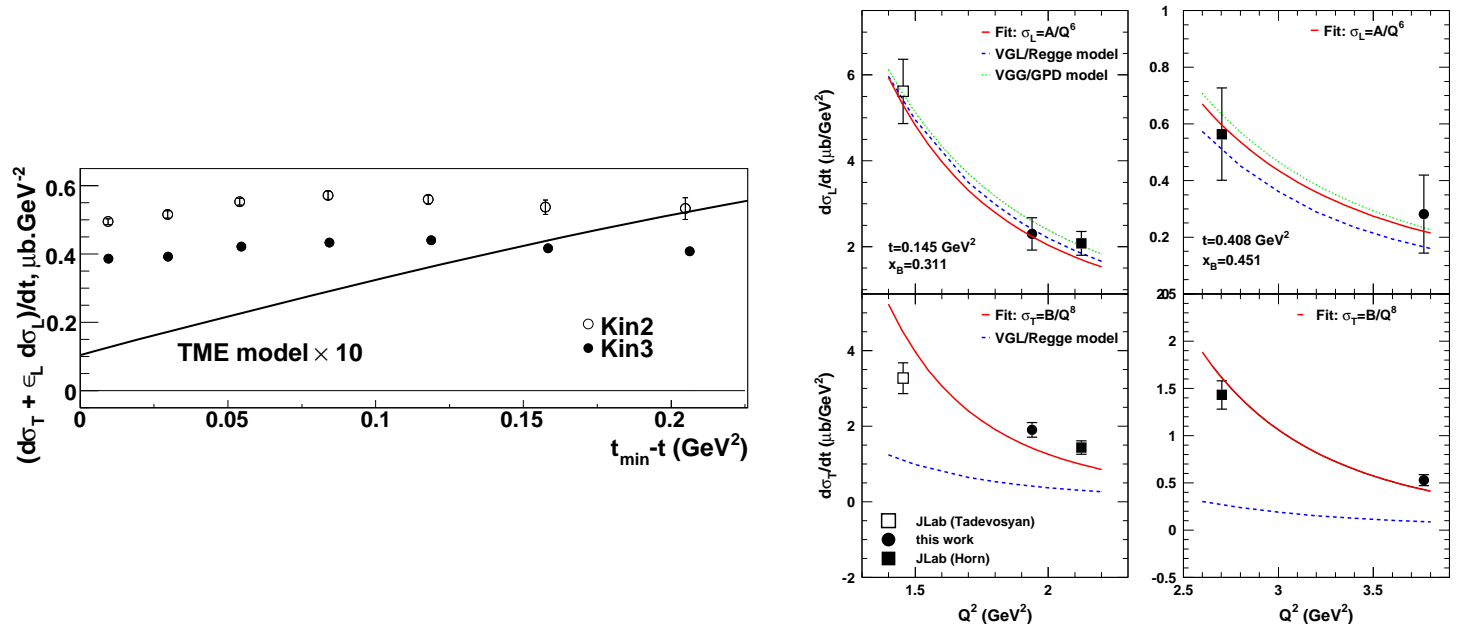

Figure 9: Cross sections for exclusive $\pi^{0}$ production measured in Hall A (left) and $\pi^{+}$production in Hall C (right). The $\pi^{0}$ measurements have not isolated the longitudinal contribution to the electroproduction cross section, so are not able to make quantitative statements regarding the applicability of soft-hard factorization. The Hall $\mathrm{C}$ results show the separated longitudinal and transverse cross sections at two values of $x$ and $t$. While the $Q^{2}$ dependence of $\sigma_{L}$ is consistent with the $Q^{-6}$ expectation, the precision is not sufficient to make strong statements about factorization.

\subsection{Pseudoscalar Meson Production}

A complete GPD program necessarily requires the measurement of other final states in addition to the photon. Measurements of Deep Exclusive meson production are a bit more challenging to interpret theoretically, however, as the hadronic structure of the meson leads to larger higher order corrections. In addition, factorization in meson production has been proven only for the part of the cross section coming from the longitudinal virtual photon, thus requiring a Rosenbluth type separation, or other technique to isolate the longitudinal response.

Fig. 9 (left) shows initial results for the cross section in Deep Exclusive $\pi^{0}$ production in Hall A [19]. While it was not possible to isolate the longitudinal cross section, $\sigma_{L}$, in this experiment, the unseparated cross sections already provide much information of interest. The apparently small $Q^{2}$ dependence suggests (although does not prove) that the process is not dominated by the longitudinal cross section, or that $\sigma_{L}$ does not follow the $Q^{-6}$ dependence one expects if soft-hard factorization applies. A follow-up experiment scheduled to run in the fall of 2010 in Hall A will allow a separation on the longitudinal and transverse contributions to the $\pi^{0}$ cross section [20]. Hall $\mathrm{B}$ has also extracted preliminary cross sections for $\pi^{0}$ production, and are in the process of finalizing their analysis before publication.

Results for exclusive $\pi^{+}$production are also shown in Fig. 9 (right) [21]. Here, the longitudinal and transverse cross sections were extracted and the $Q^{2}$ dependence at fixed $x$ and $-t$ was studied to test the $Q^{-6}$ dependence of $\sigma_{L}$ that is expected if soft-hard factorization applies. While the results for $\sigma_{L}$ are consistent with this expectation, the precision is not yet sufficient to make very strong statements about factorization. An experiment planned for after the $12 \mathrm{GeV}$ Upgrade will revisit this issue with larger $Q^{2}$ reach and improved precision [22]. 


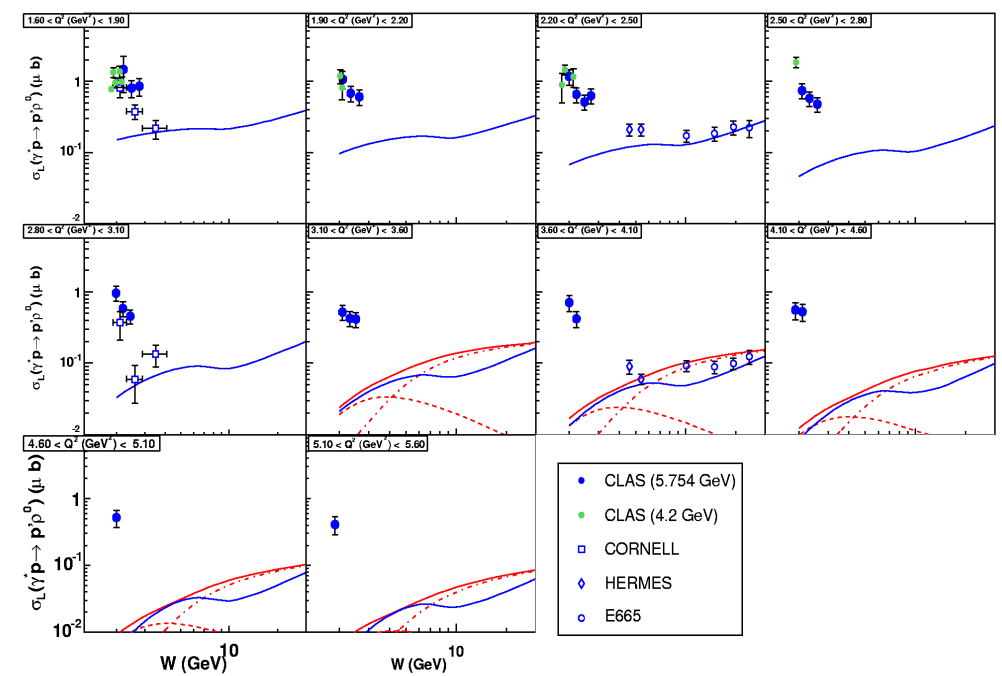

Figure 10: Longitudinal cross sections for exclusive $\rho^{0}$ production from Hall B. The curves are calculations for the cross section calculated using various GPD parametrizations. Similar data for $\omega, \phi$, and $\rho^{+}$also exist.

\subsection{Vector Meson Production}

Deep exclusive vector meson production has also been studied extensively at Jefferson Lab, primarily in Hall B. Results for $\rho^{0}$ production are shown in Fig. 10; here the longitudinal cross section has been isolated using the angular distribution of the decay products of the electro-produced vector mesons [23]. Data also exist for $\omega, \phi$, and $\rho^{+}$production. A primary conclusion from these data is that agreement with what has been considered "standard" GPD models is rather poor, better agreement being found with Regge based calculations, or GPD models with large higher order corrections.

\section{Summary}

Jefferson Lab has a large and varied scientific program providing information on the partonic structure of hadrons via many avenues. The high luminosity available allows access to relatively rare processes, in particular Deep Exclusive reactions and semi-inclusive processes at large transverse momenta. Although not discussed in a great deal of depth here, the high polarization of the Jefferson Lab electron beam, combined with the ever-improving polarized target technology allows access to spin observables with excellent precision. The Jefferson Lab $12 \mathrm{GeV}$ Upgrade, both the increased beam energy and the new equipment and spectrometers in the experimental halls, will give unprecedented access to nucleon structure at large $x$.

\section{Acknowledgments}

I would like to thank the many Jefferson Lab scientists who contributed plots and information for this presentation. In particular, comments from Hugh Montgomery and Rolf Ent were especially helpful. 
This work was supported by DOE contract No.DE-AC05-06OR23177, under which Jefferson Science Associates, LLC (JSA) operates the Thomas Jefferson National Accelerator Facility.

\section{References}

[1] J. Alcorn et al., Nucl. Instrum. Meth. A 522, 294 (2004).

[2] B. A. Mecking et al. [CLAS Collaboration], Nucl. Instrum. Meth. A 503, 513 (2003).

[3] A. Accardi, M. E. Christy, C. E. Keppel, P. Monaghan, W. Melnitchouk, J. G. Morfin and J. F. Owens, Phys. Rev. D 81, 034016 (2010) [arXiv:0911.2254 [hep-ph]].

[4] J. Seely et al., Phys. Rev. Lett. 103, 202301 (2009) [arXiv:0904.4448 [nucl-ex]].

[5] P. Solvignon, D. Gaskell and J. Arrington, AIP Conf. Proc. 1160, 155 (2009) [arXiv:0906.0512 [nucl-ex]].

[6] J. P. Chen, arXiv:1001.3898 [nucl-ex].

[7] V. A. Sulkosky, AIP Conf. Proc. 1155, 93 (2009).

[8] M. Amarian et al. [Jefferson Lab E94-010 Collaboration], Phys. Rev. Lett. 92, 022301 (2004) [arXiv:hep-ex/0310003].

[9] P. Solvignon, AIP Conf. Proc. 1155, 101 (2009) [arXiv:0906.0928 [nucl-ex]].

[10] F. R. Wesselmann et al. [RSS Collaboration], Phys. Rev. Lett. 98, 132003 (2007) [arXiv:nucl-ex/0608003].

[11] T. Navasardyan et al., Phys. Rev. Lett. 98, 022001 (2007) [arXiv:hep-ph/0608214].

[12] H. Mkrtchyan et al., Phys. Lett. B 665, 20 (2008) [arXiv:0709.3020 [hep-ph]].

[13] H. Avakian et al. [The CLAS Collaboration], arXiv:1003.4549 [hep-ex].

[14] M. Anselmino, A. Efremov, A. Kotzinian and B. Parsamyan, Phys. Rev. D 74, 074015 (2006) [arXiv:hep-ph/0608048].

[15] C. Munoz Camacho et al. [Jefferson Lab Hall A Collaboration and Hall A DVCS Collaboration], Phys. Rev. Lett. 97, 262002 (2006) [arXiv:nucl-ex/0607029].

[16] F. X. Girod et al. [CLAS Collaboration], Phys. Rev. Lett. 100, 162002 (2008) [arXiv:0711.4805 [hep-ex]].

[17] S. Chen et al. [CLAS Collaboration], Phys. Rev. Lett. 97, 072002 (2006) [arXiv:hep-ex/0605012].

[18] M. Mazouz et al. [Jefferson Lab Hall A Collaboration], Phys. Rev. Lett. 99, 242501 (2007) [arXiv:0709.0450 [nucl-ex]].

[19] E. Fuchey et al. [Jefferson Lab Hall A Collaboration], [arXiv:nucl-ex/1003.2938].

[20] Jefferson Lab Experiment 07-007, "Complete Separation of Deeply Virtual Photon and $\pi^{0}$ Electroproduction Observables of Unpolarized Protons."

[21] T. Horn et al., Phys. Rev. C 78, 058201 (2008) [arXiv:0707.1794 [nucl-ex]].

[22] Jefferson Lab Experiment 12-07-105, "Scaling Study of the L-T Separated Pion Electroproduction Cross Section at $11 \mathrm{GeV}$."

[23] S. A. Morrow et al. [CLAS Collaboration], Eur. Phys. J. A 39, 5 (2009) [arXiv:0807.3834 [hep-ex]]. 